\title{
Optimization approach to unified AC/DC power flow applied to traction systems with catenary voltage constraints
}

\author{
M. Coto, P. Arboleya*, C. Gonzalez-Moran \\ University of Oviedo, Electrical Engineering Departament
}

\begin{abstract}
This paper presents two innovative contributions related to the combined $\mathrm{AC} / \mathrm{DC}$ power flow in railway power supply systems (RPSS). First, most of the power flow equations (the linear ones) are expressed in a compact matrix form by using graph theory based protocol. Such approach simplifies the statement of the unified power flow problem and allows the train motion to be modeled without varying the system topology. Second, the problem is formulated as an Optimization Problem (OP) instead of using the non-constrained power flow approach. This technique allows the authors to simulate the effect of trains regenerative braking, considering system constraints such us the catenary voltage limit, which determines the amount of available regenerated energy injected to the network, and burned through the resistors.
\end{abstract}

Keywords: Reversible substations, Regenerative braking, Load Flow,

\footnotetext{
${ }^{*}$ Corresponding author

Email address: cotomanuel@uniovi.es, arboleyapablo@uniovi.es, gonzalezmorcristina@uniovi.es (M. Coto, P. Arboleya*, C. Gonzalez-Moran)
} 
Railways, Traction Power Systems

1 Nomenclature

2 Variables

3 $\Gamma \quad$ Incidence matrix.

${ }_{4} \mathbf{R}, \mathbf{X} \quad$ Resistance and reactance matrices.

$5 \mathbf{v}, \mathbf{i} \quad$ Voltage and current vectors.

6 I, S Identity and block diagonal matrices.

7 M Linear equations matrix.

$8 \mathbf{z} \quad$ Current and voltage solution vector.

9 P, Q Active and reactive power matrices.

10 Number of.

${ }_{11} R_{e q i} \quad$ Converter equivalent resistance.

12 Superscripts

${ }_{13} T$ Transpose matrix.

${ }_{14} D C \quad$ DC system.

${ }_{15} A C \quad$ AC system.

${ }_{16} L \quad$ Links.

${ }_{17}$ Subscripts

${ }_{18} t, s \quad$ Train, substation.

$19 \quad d, q \quad$ Direct and quadrature components.

${ }_{20} B, N \quad$ Branch, node.

${ }_{21} i, j \quad$ ith and jth element. 


\section{Introduction}

Modern electric locomotive units include regenerative braking mainly for three reasons. The first one is the energy saving when a train injects part of the braking kinetic energy into the electrical grid, to be consumed by a nearby powering train or returned to the AC system through a reversible substation. The second one is a security reason. Pneumatic braking system can not cover long distances with long gradients and it must be combined with some kind of electrical braking. In addition, the use of regenerative breaking instead other electric braking systems, prevents from the tunnel temperature rising in underground railways [? ], minimizing energy consumption in airconditioning or ventilating equipments.

A common situation nowadays is the use of modern units in old RPSS. In these systems, the energy injected into the system by a train when it is braking, must be consumed by other trains plus some electrical losses, because in networks with no reversible substations, the energy can not flow upstream through the non-controlled rectifiers. If the available regenerated braking energy is greater than the demanded energy, the train must activate the rheostatic braking when the catenary voltage reaches a given value (for instance, $1800 \mathrm{~V}$ for $1500 \mathrm{~V}$ RPSS).

In this situation, it is necessary to develop $\mathrm{AC} / \mathrm{DC}$ combined power flow methods, considering the use of regenerative units in DC traction networks with catenary voltage constraints and no reversible substations.

Two main trends for computing the power flow in AC-supplied DC traction systems are reported in the literature. The unified method introduced in [? ] and improved in [? ? ? ] simultaneously solves the whole sys- 
tem AC/DC equations. This method has also been called extended variable method, because the $\mathrm{DC}$ variables are added to the $\mathrm{AC}$ solution vector. The main drawback of this sort of methods is that they are very hard to program [? ]. This disadvantage was overcame in [? ] applying the graph theory and matrix formulation to the problem statement. However, in the cited work the possibility of including constraints to the problem has not been considered.

The second trend is the sequential method. It was proposed in [? ] and evolved in [? ? ? ? ]. It applies an iterative procedure between AC and DC systems. This method considers AC/DC converters as voltage or current sources from the DC subsystem point of view, and loads from the AC point of view. In most of the cases, a plain voltage profile in the DC subsystem is assumed in all DC nodes. Under this assumption, the power demanded by each substation from the AC system is computed. Thereafter the AC powerflow is solved to correct the initial DC voltage profile. The main advantage of sequential methods lies in the simplicity of implementation, however they present some convergence problems [? ].

In most of the above described works, the trains are always electrical loads and the problem of the catenary voltage rise in case of the regenerative braking is not considered. First works proposing power flows in DC traction networks with unidirectional substations and constrained voltages in catenaries during regenerative braking were developed in [? ? ? ]. Modeling regenerative braking with this kind of traditional methods is possible, but it requires an iterative process because the available power can not be the final regenerated power due to the catenary voltage constraints. An initial regenerated power must be supposed and corrected in successive iterations. 
With the proposed approach the final regenerated power is obtained avoiding this iterative process.

In [? ? ] only the DC subsystem problem is considered. The AC/DC substations are assumed as DC voltage or current sources, with a series or parallel connected resistance respectively. The use of this approach, however, does not consider the effect of the AC grid voltage drops in the DC subsystem. Thus, in a real scenario two identical AC/DC converters with the same load level and different voltage outputs can be found [? ]; but with the above described methods such situation cannot be modeled.

In [? ] a combined AC/DC load flow based on a sequential approach using the Gauss-Seidel method is proposed. In this case, the effect of the $\mathrm{AC}$ network can be simulated but the iterative process to obtain the power injected by the trains is similar to the previous described.

In $[?]$, the authors study the effect of the bidirectional substations in the DC voltage profile considering units with regenerative braking. The sequential approach is adopted to solve the combined AC/DC power flow. However, in this case, no voltage constraints are considered and the authors just compare the obtained voltages with and without reversible substations.

This work presents the next innovative contributions when compared with previous work:

- A Graph theory based method to describe the AC/DC electrical system and the space-time variation of the loads (trains) is developed [? ]. The use of graph theory to describe, analyze and solve power systems is not new, but it is still in vogue [? ? ? ]. In the present work, the authors have used such theory to propose the systematic statement of 
the equations based on a matrix formulation and allowing the use of sparsity techniques that reduce the computational time.

- It also combines the unified power flow approach with the regenerative braking of the units but considering the system constraints (in this case the catenary voltage).

- Due to the problem constraints, in case of regenerative braking, sometimes the injected power is less than the available regenerated power, and part of this regenerated power must be burned in the rheostatic brakes. The statement of the problem as an optimization problem, permit us to make the injected power calculation without the need of an iterative process.

The authors have used the widely accepted stationary equivalent method for moving loads proposed in [? ]. This method assumes that the train speed is not so high as to induce pronounced electrical transients, and that the DC traction network evolves slowly from one state to another as the locations and the trains input power vary. Using this stationary equivalent, temporal analyses of RPSS are computed by solving successive time instants.

The paper will be structured as follows; In section 2, the problem statement will be described. First, a general overview of the problem will be given and then we will explain the proposed method to describe the AC/DC topology, the movement of the trains and the system constraints. In section 3 a set of cases of study are presented and validated by using a commercial software (DIgSILENT). Once the instantaneous results are validated, the same procedure is applied to the Vitoria Tram case (city located in the north of 
Spain). Finally, the conclusions are presented in section 4.

\section{Problem Statement}

The combined AC/DC power flow will be stated as an OP [? ? ]. This will allow us to study the effect of the regenerative braking over the network, considering no bidirectional substations and constrained catenary voltages without the need of an iterative procedure. The mathematical formulation will be expressed as follows:

$$
\begin{aligned}
\min & f(\mathbf{z}) \\
\text { subject to } & g(\mathbf{z})=0 \\
& l(\mathbf{z}) \leq 0 \\
& \mathbf{z}_{\min } \leq \mathbf{z} \leq \mathbf{z}_{\max }
\end{aligned}
$$

Where:

- $\mathbf{z}$ is a vector of unknowns with lower and upper limits, $\mathbf{z}_{\min }$ and $\mathbf{z}_{\max }$ respectively. It contains all network currents and voltages. In the DC part, the variables are the branch currents, the currents absorbed or injected by the trains, the currents in the DC part of the links, which connect the $\mathrm{AC}$ and the DC subsystems, and the voltages in all DC nodes, including substations and trains. Regarding the AC subsystem, the vector $\mathbf{z}$ contains all node voltages and branch currents in $d q$ components.

- $f(\mathbf{z})$ is a scalar function modeling the power demanded by all trains. The roll that this function plays, is making the trains to inject the 
maximum power without exceeding the maximum permitted catenary voltage during a regenerative braking process.

- $g(\mathbf{z})$ is a set with all equations needed to solve the power flow. They can be divided into two subsets. The first one contains Kirchhoff Current and Voltage laws (KCL and KVL), these equations are linear and they are expressed in a compact matrix form as it will be explained below. The second one is a set of non-linear equations modeling the $P Q$ and $P V$ nodes of the AC subsytem, the converters of the links between the $\mathrm{AC}$ and the DC subsystems and the trains in traction mode.

- $l(\mathbf{z})$ is a set of non linear inequalities modeling two processes. The former is the trains behavior when they are regenerating energy during the braking process. The latter forces the unidirectional power flow in the rectifiers connecting the $\mathrm{AC}$ and the DC subsystem.

In the following subsections, the prosed formulation will be extended. In tables 9 and 10, all vectors and matrices used in the proposed formulation with their dimensions can be observed.

\subsection{Objective Function}

We have defined the objective function $f(\mathbf{z})$ as the sum of demanded power by all trains. It can be expressed as follows:

$$
f(\mathbf{z})=\sum_{i=1}^{n_{t}} P_{t i}=\sum_{i=1}^{n_{t}} v_{N i}^{D C} i_{t i}^{D C}=0
$$

Previous works solved this kind of problem by using an iterative process. In such process, an initial value of trains injected power was assumed. Then 
the catenary voltage was calculated by means of a traditional power flow approach and if it exceed its maximum, the initial value was corrected. This procedure was repeated until convergence.

With the proposed OP approach, the solution will be the one permitting the maximum power injected by the trains, with no catenary voltage constraint violation.

\subsection{Power Flow Equations}

All linear equations are stated in a compact matrix form based in graph theory. The proposed method uses the node incidence matrix $\boldsymbol{\Gamma}$ to obtain such equations simplifying the implementation procedure when compared with the traditional one. The use of $\boldsymbol{\Gamma}$ for summarizing the network topology is well known in graph theory [? ]. The $\boldsymbol{\Gamma}$ rows and columns will represent respectively the graph edges (lines or branches in our case) and nodes (substations). The $\boldsymbol{\Gamma}_{i j}$ elements are defined as follows:

- $\boldsymbol{\Gamma}_{i j}=1$ when positive current in branch $i$, leaves node $j$.

- $\boldsymbol{\Gamma}_{i j}=-1$ when positive current in branch $i$, flows towards node $j$.

- $\boldsymbol{\Gamma}_{i j}=0$ when no connection exists.

By using the $\boldsymbol{\Gamma}$ matrix, all equations representing Kirchhoff's Voltage and Current Laws, can be expressed in a compact form as follows:

$$
g(\mathbf{z})=\mathbf{M} \mathbf{z}^{T}=0
$$

Where $\mathbf{z}$ is the vector representing voltage and current magnitudes that is formed as follows:

$$
\begin{aligned}
& \mathbf{z}=\left[\begin{array}{lllllll}
\mathbf{i}_{B}^{D C} & \mathbf{i}_{B d}^{A C} & \mathbf{i}_{B q}^{A C} & \mathbf{i}_{t}^{D C} & \mathbf{i}_{B}^{L} & \mathbf{i}_{B d}^{L} & \ldots
\end{array}\right. \\
& \left.\begin{array}{lllllllll}
\ldots & \mathbf{i}_{N d}^{A C} & \mathbf{i}_{B q}^{L} & \mathbf{i}_{N q}^{A C} & \mathbf{v}_{N}^{D C} & \mathbf{v}_{N d}^{A C} & \mathbf{v}_{N q}^{A C} & ]
\end{array}\right]
\end{aligned}
$$


The construction of $\mathbf{M}$ is represented in expression (5),where:

- $\Gamma^{D C}$ and $\Gamma^{A C}$ represent the DC and AC subsystems topology respectively, defining a constant index for each line and each node. The AC topology will remains constant so $\Gamma^{A C}$ represents all real connections in the AC subsystem. However, $\Gamma^{D C}$ represents all possible connections in the DC subsystem. For instance, a given train will be connected to all trains and all substations in the $\Gamma^{D C}$. Then, only the actual connections will be activated for a given instant by means of $\mathbf{R}_{B}^{D C}$.

- $\mathbf{R}_{B}^{D C}$ is the branch resistance matrix of the DC subsystem. It is a diagonal matrix and $r_{i i}$ represents the resistance of branch $i$. As it was mentioned, $\Gamma^{D C}$ generates a set of DC lines that are not simultaneously active at the same simulation step. The use of this formulation permits us to assign an infinite value to those inactive lines, so they do not have any influence in the system. $\mathbf{R}_{B}^{D C}$ is the only matrix affected by the train motion, and must be updated when the train changes its location. At each simulation instant, the position of each train must be read. Then, the value $r_{i i}$ must be set considering this position and the type of catenary. An infinite value must be assigned to non-active lines. For instance, in Figure 1, the branch $b 14$ is a non active branch that connects nodes 4 and 6 when there is no train between them. The element $r_{14,14}$ is set to infinite $\left(10^{6}\right)$. In this case, the resistance of branches $b 10$ and $b 12$ must be updated at each iteration, containing the information about the total resistance between the substations 4 and 6 and the train 3 respectively. If the train 3 arrives to 6 to be positioned then between 6 and 5 , the branch $b 10$ will be deactivated 
and the train 3 will be connected to 6 and 5 through the branches $b 12$ and $b 11$. Considering that trains 1 and 2 are still between substations 4 and 5, the branch $b 14$ that connects substations 4 and 6 will be activated updating $r_{14,14}$ with the value of the resistance between the above-mentioned substations.

- $\mathbf{R}_{B}^{A C}$ and $\mathbf{X}_{B}^{A C}$ are the resistance and reactance matrices respectively, representing the impedance between AC nodes. They are diagonal matrices, where $r_{i i}$ and $x_{i i}$ represent the resistance and reactance of branch $i$ respectively, or the short circuit resistance and reactance of the transformer placed in the branch, in case of the $\mathrm{AC}$ branches that connect the $\mathrm{AC}$ and the DC systems.

- I identity matrix.

- $\mathbf{S}$ is a block diagonal matrix. The first block is an identity matrix with dimensions $\left(n_{t}, n_{t}\right)$. The second block is a diagonal matrix denoted as $\mathbf{S}_{\left(n_{s}, n_{s}\right)}^{L}$. Element $s_{i i}$ belonging to $\mathbf{S}^{L}$ is 1 if the DC substation $i$ is connected to the AC network and $s_{i i}$ is 0 when the DC substation $i$ is not connected to the AC grid.

All vector and matrix dimensions are listed in tables 9 and 10 .

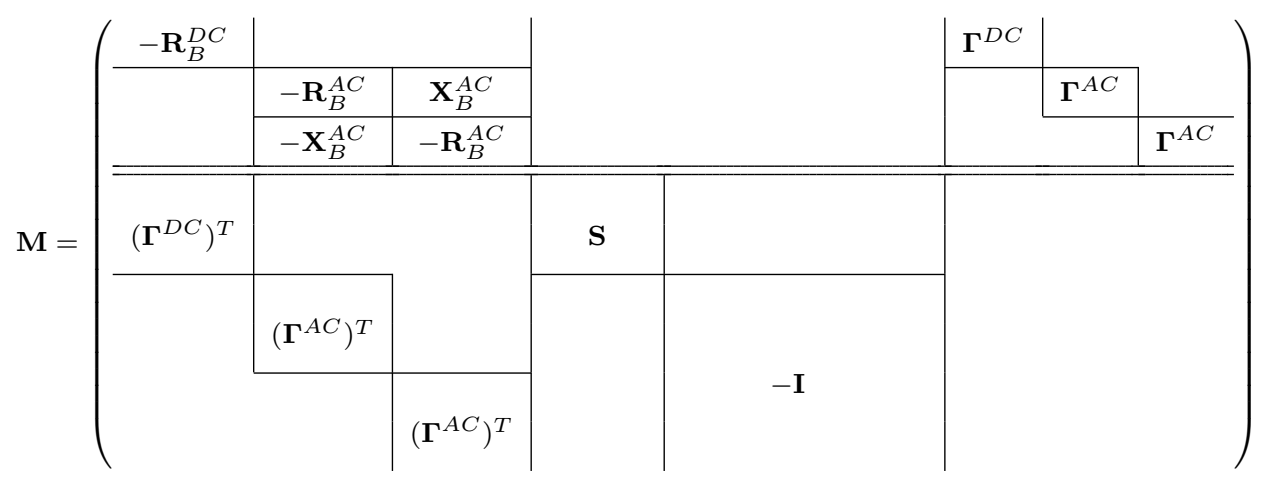


To complete the construction of $g(\mathbf{z})$, equations derived from AC network PQ and PV nodes models, train model and converter model must be added. $\mathrm{PQ}$ nodes in the $\mathrm{AC}$ network contribute with the next expressions:

$$
g(\mathbf{z})=\left\{\begin{array}{l}
v_{N d i}^{A C} i_{N d i}^{A C}+v_{N q i}^{A C} i_{N q i}^{A C}-P_{i}=0 \\
v_{N q i}^{A C} i_{N d i}^{A C}-v_{N d i}^{A C} i_{N q i}^{A C}-Q_{i}=0
\end{array}\right.
$$

The equations corresponding to the PV nodes are:

$$
g(\mathbf{z})=\left\{\begin{array}{l}
v_{N d i}^{A C} i_{N d i}^{A C}+v_{N q i}^{A C} i_{N q i}^{A C}-P_{i}=0 \\
\sqrt{\left(v_{N d i}^{A C}\right)^{2}+\left(v_{N q i}^{A C}\right)^{2}}-\left|v_{N i}^{A C}\right|=0
\end{array}\right.
$$

The following equations correspond to a simple model of an AC/DC 6 pulse diode converter:

$$
g(\mathbf{z})=\left\{\begin{array}{l}
v_{N d i}^{L} i_{B d i}^{L}+v_{N q i}^{L} i_{B q i}^{L}-v_{N i}^{L} i_{B i}^{L}=0 \\
v_{N q i}^{L} i_{B d i}^{L}-v_{N d i}^{L} i_{B q i}^{L}=0 \\
v_{N i}^{L}-1.35 \sqrt{\left(v_{N d i}^{L}\right)^{2}+\left(v_{N q i}^{L}\right)^{2}}-R_{e q i} i_{B i}^{L}=0
\end{array}\right.
$$

Where $R_{\text {eqi }}$ is the equivalent resistance of the conversion unit in the regular commutation range. Further details can be obtained from [? ? ]. By using the same procedure, complex models of non-controlled or controlled converters may be implemented. See for instance [? ? ].

Unlike other authors that develops their own software for the train simulation [? ], in our case, the train power will be provided by a software package that uses the rail and train parameters for a given unit and route developed by CAF Company. The output data of this software is the power absorbed or regenerated by the train in the DC network at each instant. This software package considers only mechanical aspects, obtaining the desired electrical power in traction mode or the available power in regenerative braking mode. 

proposed method.

Depending on the accelerating or braking state, different expressions will be used. So when train is consuming energy the equations are:

$$
g(\mathbf{z})=P_{i}-v_{N i}^{D C} i_{t i}^{D C}=0
$$

When the train is braking:

$$
l(\mathbf{z})=\left\{\begin{array}{l}
P_{i}-v_{N i}^{D C} i_{t i}^{D C} \leq 0 \\
i_{t i}^{D C} \leq 0
\end{array}\right.
$$

The last inequalities guaranty the unidirectional flow through the noncontrolled rectifiers from AC to DC subsystem.

$$
l(\mathbf{z})=i_{B i}^{L} \leq 0
$$

The voltage constrains in catenaries are set using $\mathbf{z}_{\min }$ and $\mathbf{z}_{\max }$.

\section{Results Analysis}

In this section we first study a set of given cases of an specific $\mathrm{AC} / \mathrm{DC}$ network. To validate the method, the obtained results are compared with those obtained using a commercial software (DIgSILENT), and with those obtained by solving the described set of equations using the classical procedure without the optimization approach. Then, the method presented here is applied to a real case. 


\subsection{Validation}

The system used to validate the method is depicted in Figure 1. The $\mathrm{AC} / \mathrm{DC}$ system is composed of a 6 nodes $\mathrm{AC}$ subsystem, one generator, two loads and three connections to the DC subsystem. The DC subsystem is composed by three substations and three trains, two of them in the same line. The proposed enumeration criteria is the next; we first enumerate the trains (nodes 1-3) and then the DC nodes (nodes 4-6). When a connection between the DC and AC subsystem is activated, a new auxiliary AC node is included, in this case nodes 7,8 and 9. Finally we assign numbers to AC nodes (nodes 10 to 15 ). In Figure 1, only active branches given by the real positions of the trains within the DC network, are represented.

The resistance $(R)$ and reactance $(X)$ of the AC subsystem lines are respectively $0.09962 \Omega / \mathrm{km}$ and $0.51442 \Omega / \mathrm{km}$. The AC network has 6 branches with different lengths. The lengths of these branches and the total resistance and reactance appear in Table 3.

In the $\mathrm{AC}$ network, there exist different types of nodes. Nodes 14 and 15 are PQ type and node 10 is a slack bus (see table 4). Nodes 11-13 are connection nodes with the DC subsystem.

The AC/DC links are composed by one transformer and one rectifier. In the case of study, the system has three links with the same rectifier and transformer. The rectifier is a six-pulse non-controlled type. Power transformer characteristics are summarised in Table 5.

Five different cases will be analyzed. The trains relative position will not be modified in any case. The distance between trains, between trains and substations and the power demanded by trains will be varied. All cases are 
defined in Tables 6 and 7 .

In the DC subsystem, all the catenaries are $C R 160$ type with a resistance of $0.051 \Omega / \mathrm{km}$, the rails are $54 \mathrm{Kg} / \mathrm{m}$ type with a resistance of $0.007 \Omega / \mathrm{km}$. In this case, we suppose that each train has a perfect connection to ground so the rail resistance is added to the catenary resistance. Table 6 defines, for all cases, the length of the DC branches and the resistances due to the train positions.

In Table 7 the power demanded by each train is shown. Trains 1 and 2 are always consuming energy. On the other hand, train 3 is always braking, so it injects power into DC system. In the column labelled as Ref, the available regenerated power is presented. In columns $\operatorname{Pr}$ and $D S$ the final injected powers obtained using the proposed method and the DIgSILENT software are presented respectively. The authors have also added a column labelled as NO. In this column, the described set of equations are solved by means of a classical procedure without using the optimization approach. In table 1 , the active power through unidirectional substations is presented for all cases and all methods.

As it can be observed, only in case 1, when the injected power is very low compared to the total demanded power, the three methods give the same results. In case 2 , the $\operatorname{Pr}$ and $D S$ methods show the same results. Using both methods the injected power by train 3 is used to feed trains 1 and 2 . The solution obtained by $N O$ method is different. In this case, the injected power is lower and the DC subsystem demands a higher amount of energy through the substations, as it can be observed in table 1 . In cases $(3,4$ and 5$)$ the solutions given by $\operatorname{Pr}$ and $D S$ are nearly the same. In such cases the injected 
power does not reach the maximum available. In cases 3 and 5 , the catenary voltage level arises to maximum $(1800 \mathrm{~V}$ ) (see table 2$)$. In case 4 , the injected power by the train 3 satisfies the demand of rest of trains without reaching the maximum voltage. The solution reached by $N O$ method for cases 3,4 and 5 , shows a lower power injection. In table 1 it can be observed that the demanded power through the substations using $N O$ method is always greater than the one obtained using $\operatorname{Pr}$ and $D S$.

All node voltages are shown in Table 2. In this table, the obtained RMS voltages using the proposed method can be compared with those obtained using the commercial software. It can be observed the high level of accuracy obtained with the proposed method when compared to results obtained with DIgSILENT. On contrary, the voltages profile obtained with the NO method is always lower, except for the case 1 where all the methods match up. Furthermore, it can be observed that when $N O$ is used, the catenary maximum voltage is never reached.

\subsection{Vitoria Tram Case of Study}

The application of the proposed method on a real tram line is reported in this section, showing the main simulation results during 1 hour and 30 minutes of study time.

Vitoria tram system includes two lines: Line Ibaiondo - Angulema and line Abetxuko-Angulema. The power system network is composed by two substations located in Landaberde and Angulema, as is shown in Figure 2. Each substation connects the $1648 \mathrm{~V}$ nominal voltage DC network to the AC grid by 6 pulse diode bridge rectifiers. The units used in this tram are Urban2 type from CAF manufacturer. The train power has been provided 


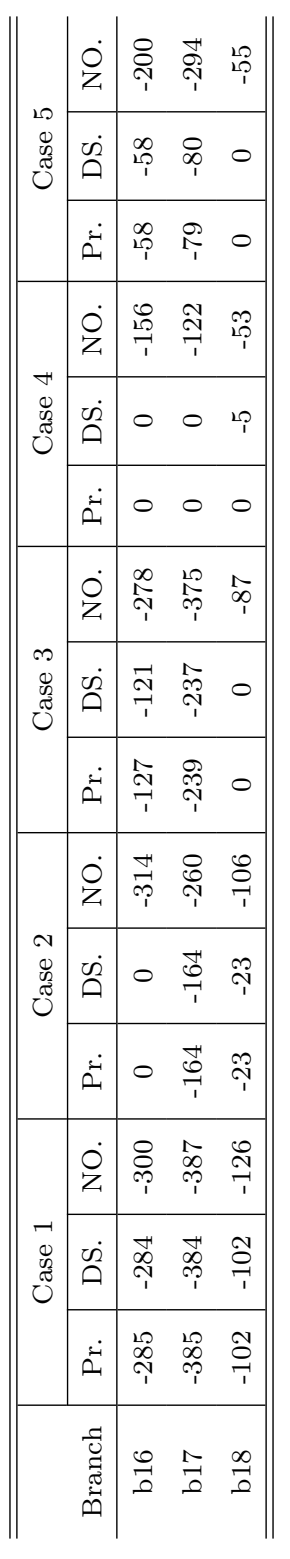

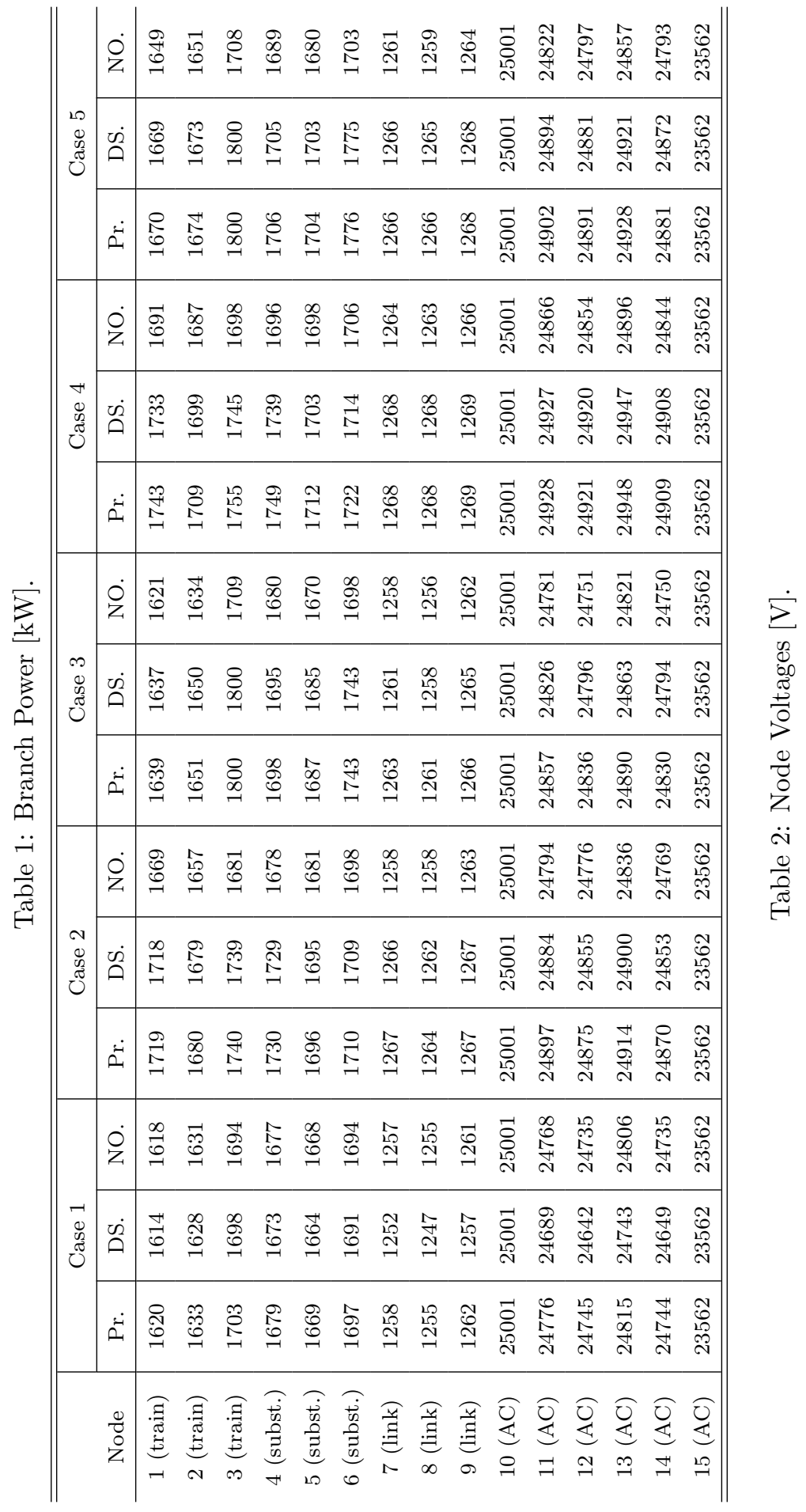


by a complex software package that uses the rail and trains parameters for a given unit and route. The output data of this software is the mechanical power demanded during the traction or available during regeneration braking by the train. The final absorbed or injected power is calculated by using the proposed approach. In Table 8 the tram timetable in the study time is shown, and results of this case are shown in figure 3. This figure presents the substation voltages and power flows. As it can be observed there are several time instants in which the voltage reaches the upper limit of 1800 $\mathrm{V}$, resulting in catenary saturation. In such time steps, part of the available regenerated power is burned in the rheostatic brake system.

\section{Conclusions}

The use of the optimization approach, has revealed to be a very useful tool for solving power flows in traction networks, specially with unidirectional non-controlled substations when the trains are equipped with regenerative braking systems. When compared the results with those obtained with the non-constrained power flow approach, more realistic voltage profiles are achieved with the proposed OP formulation. The combination of the OP formulation with the graph theory, permitted the authors to state all the equations in a really simple manner, simplifying the post-processing and result comparison of different time steps.

The method was validated through the comparison with a commercial software package (DIgSILENT), obtaining a high accuracy. Once the results were validated, the method was successfully applied to a real case (Vitoria Tram). 
336 In this paper, the formulation was applied to a specific problem, in which 337 the amount of injected power is constrained by the catenary maximum volt338 age. However, it could be applied to any constrained power flow problem. 339 The use of graph theory to state all linear equations in a compact matrix 340 form could be also extended and generalized to any other power system de341 scription. 
342 List of Figures

${ }_{343} 1$ Proposed AC/DC system. The upper part of the system cor-

344 responds to the AC subsystem and bottom the DC subsystem. 21

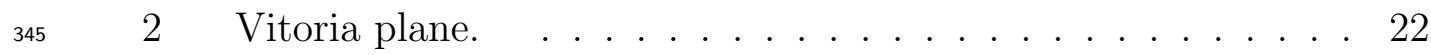

$346 \quad 3$ Voltage and Power in Angulema and Landaberde substations. 23 


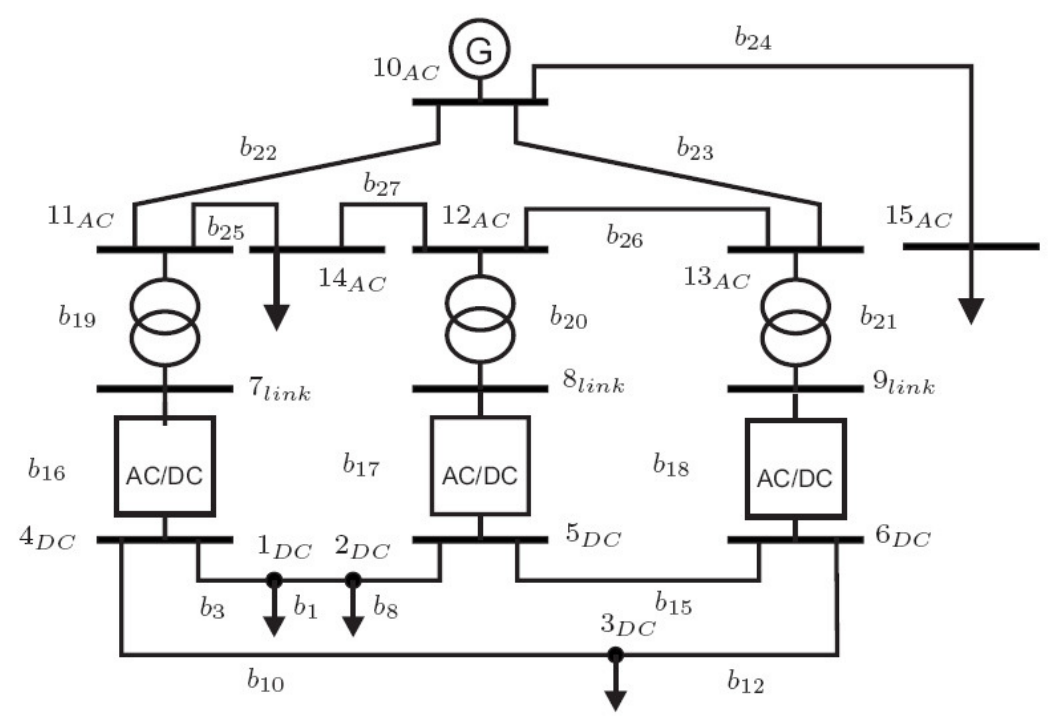

Figure 1: Proposed AC/DC system. The upper part of the system corresponds to the AC subsystem and bottom the DC subsystem. 


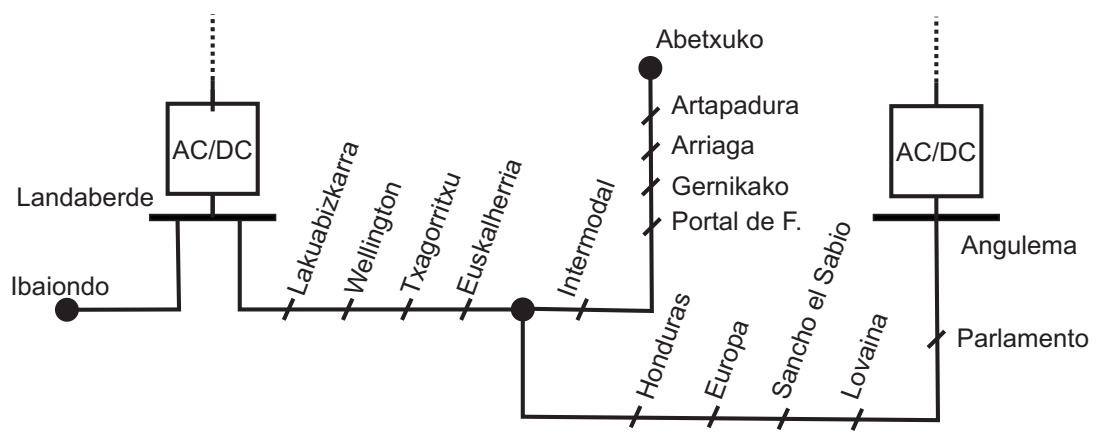

Figure 2: Vitoria plane. 

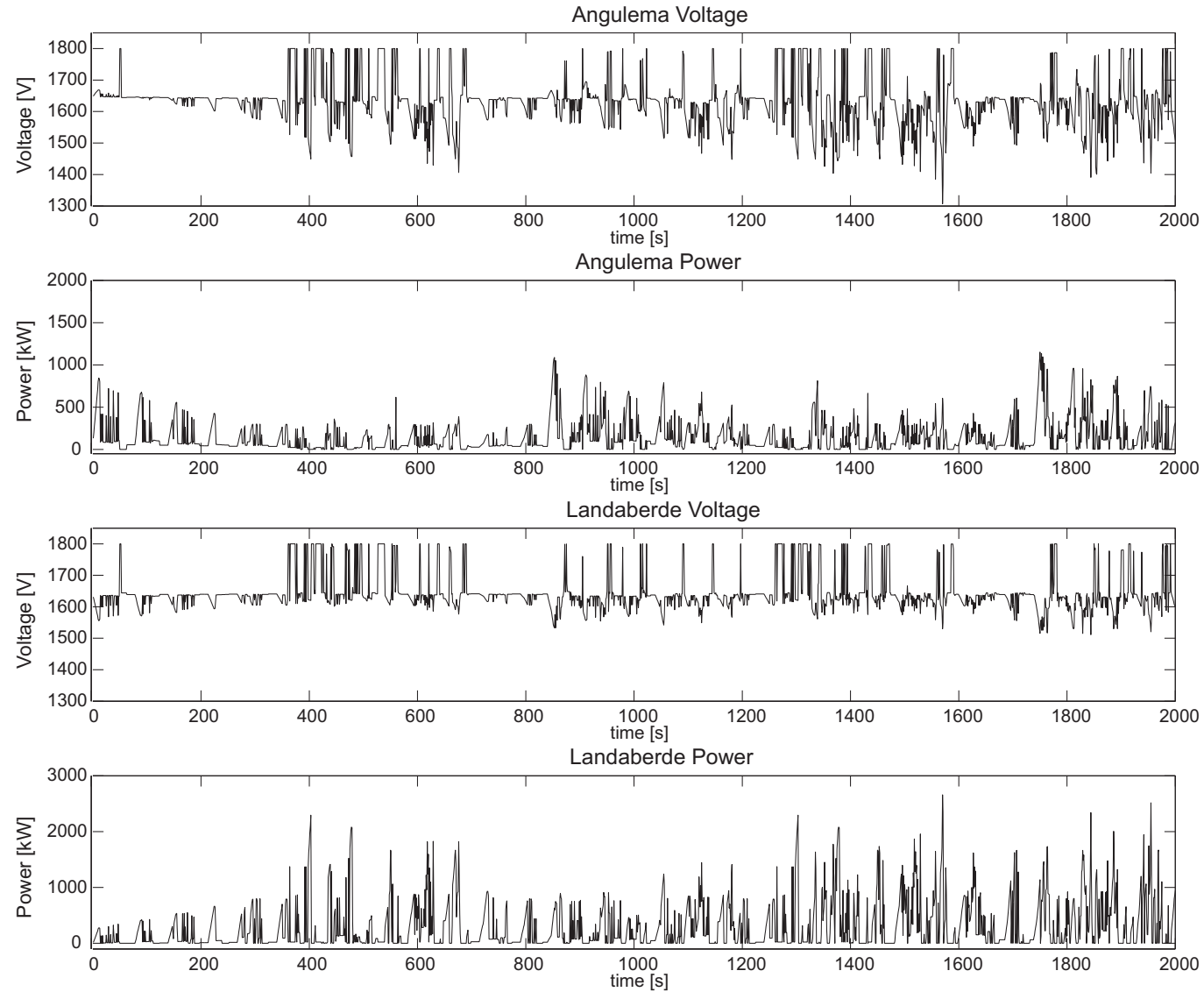

Figure 3: Voltage and Power in Angulema and Landaberde substations. 
347 List of Tables

$348 \quad 1 \quad$ Branch Power $[\mathrm{kW}] . \ldots \ldots \ldots \ldots$. . . . . . . . . . . . . . . . . . . . . . 17

$349 \quad 2$ Node Voltages $[\mathrm{V}] . \ldots \ldots \ldots \ldots \ldots$

$350 \quad 3$ AC branches electrical parameters. . . . . . . . . . . . 25

3514 AC nodes electrical parameters. . . . . . . . . . . . 26

3525 Power transformer parameters. . . . . . . . . . . . 27

${ }_{353} 6$ Branch data. . . . . . . . . . . . . . . . . . . . . 28

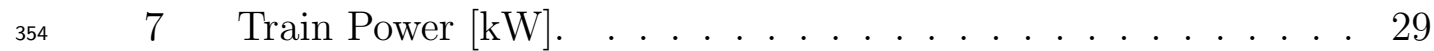

$355 \quad 8$ Vitoria tram schedule. . . . . . . . . . . . . 30

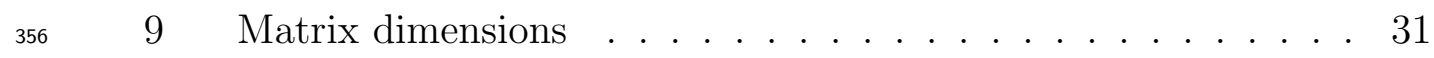

$357 \quad 10$ Vector dimensions . . . . . . . . . . . . . . . . . 32 


\begin{tabular}{c|c|c|c|c|c|c}
\hline \hline \multirow{2}{*}{ Parameter } & \multicolumn{6}{|c}{ Branch } \\
\cline { 2 - 7 } & $b_{22}$ & $b_{23}$ & $b_{24}$ & $b_{25}$ & $b_{26}$ & $b_{27}$ \\
\hline Length $[\mathrm{km}]$ & 72.4 & 77.2 & 65 & 20 & 40.2 & 19 \\
$\mathrm{R}[\Omega]$ & 7.212 & 7.691 & 6.475 & 1.992 & 4.005 & 1.893 \\
$\mathrm{X}[\Omega]$ & 37.243 & 39.712 & 33.436 & 10.288 & 20.679 & 9.774 \\
\hline \hline
\end{tabular}

Table 3: AC branches electrical parameters. 


\begin{tabular}{c|c|c|c|c}
\hline \hline Node & Type & P [MW] & Q [MVar $]$ & V [kV] \\
\hline 10 & Slack & - & - & 25 \\
14 & PQ & 0.4 & 0 & - \\
15 & PQ & 1 & 0.8 & - \\
\hline \hline
\end{tabular}

Table 4: AC nodes electrical parameters. 


\begin{tabular}{c|c}
\hline \hline Nominal Power (kVA) & 1315 \\
Nominal frequency (Hz) & 50 \\
Primary nominal voltage (V) & 24000 \\
Secondary nominal voltage (V) & 1221 \\
Connection & Delta-wye \\
Electric losses (W) & 13300 \\
Short-circuit voltage (\%) & 5.5 \\
\hline \hline
\end{tabular}

Table 5: Power transformer parameters. 


\begin{tabular}{|c|c|c|c|c|c|c|c|}
\hline & & b1 & b3 & $\mathrm{b} 8$ & b10 & b12 & b15 \\
\hline \multirow[b]{2}{*}{ Case 1} & Length $(\mathrm{km})$ & 3 & 5 & 2 & 14 & 6 & 6 \\
\hline & $\mathrm{R}(\Omega)$ & 0.177 & 0.295 & 0.118 & 0.826 & 0.354 & 0.354 \\
\hline \multirow[b]{2}{*}{ Case 2} & Length $(\mathrm{km})$ & 7.5 & 0.5 & 2 & 0.5 & 19.5 & 6 \\
\hline & $\mathrm{R}(\Omega)$ & 0.4425 & 0.0295 & 0.118 & 0.0295 & 1.1505 & 0.354 \\
\hline \multirow[b]{2}{*}{ Case 3} & Length $(\mathrm{km})$ & 3 & 5 & 2 & 14 & 6 & 6 \\
\hline & $\mathrm{R}(\Omega)$ & 0.177 & 0.295 & 0.118 & 0.826 & 0.354 & 0.354 \\
\hline \multirow[b]{2}{*}{ Case 4} & Length $(\mathrm{km})$ & 7.5 & 0.5 & 2 & 0.5 & 19.5 & 6 \\
\hline & $\mathrm{R}(\Omega)$ & 0.4425 & 0.0295 & 0.118 & 0.0295 & 1.1505 & 0.354 \\
\hline \multirow[b]{2}{*}{ Case 5} & Length $(\mathrm{km})$ & 3 & 5 & 2 & 18 & 2 & 6 \\
\hline & $\mathrm{R}(\Omega)$ & 0.177 & 0.295 & 0.118 & 1.062 & 0.118 & 0.354 \\
\hline
\end{tabular}

Table 6: Branch data. 


\begin{tabular}{l|c|c|c|c|c|c}
\hline \hline & \multirow{2}{*}{ Train 1 } & \multirow{2}{*}{ Train 2 } & \multicolumn{4}{|c}{ Train 3 } \\
\cline { 4 - 7 } & & & Ref. & Pr. & DS. & NO. \\
\hline Case 1 & 443 & 380 & -80 & -80 & -80 & -80 \\
Case 2 & 443 & 380 & -650 & -650 & -650 & -152 \\
Case 3 & 443 & 380 & -650 & -511 & -520 & -110 \\
Case 4 & 243 & 180 & -650 & -430 & -425 & -93.3 \\
Case 5 & 243 & 380 & -650 & -526 & -525 & -88.6 \\
\hline \hline
\end{tabular}

Table 7: Train Power [kW]. 


\begin{tabular}{|c|c|c|c|c|c|c|c|}
\hline Departure & From & To & Arrival & Departure & From & To & Arrival \\
\hline $6: 00: 00$ & ibaiondo & angulema & $6: 17: 20$ & $6: 52: 00$ & abetxuko & angulema & $7: 09: 00$ \\
\hline $6: 07: 00$ & abetxuko & angulema & $6: 24: 00$ & $6: 53: 00$ & angulema & abetxuko & $7: 10: 00$ \\
\hline $6: 15: 00$ & ibaiondo & angulema & $6: 32: 20$ & 7:00:00 & ibaiondo & angulema & $7: 17: 20$ \\
\hline $6: 22: 00$ & abetxuko & angulema & $6: 39: 00$ & 7:01:00 & angulema & ibaiondo & $7: 18: 20$ \\
\hline $6: 23: 00$ & angulema & abetxuko & $6: 40: 00$ & $7: 07: 00$ & abetxuko & angulema & $7: 24: 00$ \\
\hline $6: 30: 00$ & ibaiondo & angulema & $6: 47: 20$ & 7:08:00 & angulema & abetxuko & $7: 25: 00$ \\
\hline $6: 31: 00$ & angulema & ibaiondo & $6: 48: 20$ & $7: 15: 00$ & ibaiondo & angulema & $7: 32: 20$ \\
\hline $6: 37: 00$ & abetxuko & angulema & $6: 54: 00$ & $7: 16: 00$ & angulema & ibaiondo & $7: 33: 20$ \\
\hline $6: 38: 00$ & angulema & abetxuko & $6: 55: 00$ & $7: 22: 00$ & abetxuko & angulema & $7: 39: 00$ \\
\hline $6: 45: 00$ & ibaiondo & angulema & $7: 02: 20$ & $7: 23: 00$ & angulema & abetxuko & $7: 40: 00$ \\
\hline $6: 46: 00$ & angulema & ibaiondo & $7: 03: 20$ & & & & \\
\hline
\end{tabular}

Table 8: Vitoria tram schedule. 


\begin{tabular}{c|c}
\hline \hline Matrix & Dimensions \\
\hline $\boldsymbol{\Gamma}^{D C}$ & $\left(n_{B}^{D C}, n_{N}^{D C}\right)$ \\
$\boldsymbol{\Gamma}^{A C}$ & $\left(n_{B}^{A C}, n_{N}^{A C}\right)$ \\
$\boldsymbol{\Gamma}^{L}$ & $\left(n_{B}^{L},\left(n_{N}^{D C}+n_{N}^{A C}\right)\right)$ \\
$\mathbf{R}_{B}^{A C}, \mathbf{X}_{B}^{A C}$ & $\left(n_{B}^{A C}, n_{B}^{A C}\right)$ \\
$\mathbf{R}_{B}^{D C}$ & $\left(n_{B}^{D C}, n_{B}^{D C}\right)$ \\
$\mathbf{I}$ & $\left(2 n_{N}^{A C}, 2 n_{N}^{A C}\right)$ \\
$\mathbf{S}$ & $\left(n_{N}^{D C}, n_{N}^{D C}\right)$ \\
$\mathbf{M}$ & $\left(\left(n_{B}^{D C}+2 n_{B}^{A C}+n_{N}^{D C}+2 n_{N}^{A C}\right),\left(n_{B}^{D C}+2 n_{B}^{A C}+n_{N}^{D C}+4 n_{N}^{A C}+n_{N}^{D C}\right)\right)$ \\
$\boldsymbol{\Gamma}, \mathbf{P}, \mathbf{Q}$ & $\left(\left(n_{B}^{D C}+n_{B}^{L}+n_{B}^{A C}\right),\left(n_{N}^{A C}+n_{N}^{D C}\right)\right)$ \\
\hline \hline
\end{tabular}

Table 9: Matrix dimensions 


\begin{tabular}{c|c||c|c}
\hline \hline Vector & Dimensions & Vector & Dimensions \\
\hline $\mathbf{v}_{N d}^{A C}, \mathbf{v}_{N q}^{A C}$ & $\left(1, n_{N}^{A C}\right)$ & $\mathbf{i}_{B d}^{A C}, \mathbf{i}_{B q}^{A C}$ & $\left(1, n_{B}^{A C}\right)$ \\
$\mathbf{v}_{N}^{D C}$ & $\left(1, n_{N}^{D C}\right)$ & $\mathbf{i}_{N d}^{A C}, \mathbf{i}_{N q}^{A C}$ & $\left(1, n_{B}^{A C}\right)$ \\
& & $\mathbf{i}_{B d}^{L}, \mathbf{i}_{B q}^{L}$ & $\left(1, n_{B}^{L}\right)$ \\
& & $\mathbf{i}_{B}^{D C}$ & $\left(1, n_{B}^{D C}\right)$ \\
& & $\mathbf{i}_{B}^{L}$ & $\left(1, n_{s}\right)$ \\
& & $\mathbf{i}_{t}^{D C}$ & $\left(1, n_{t}\right)$ \\
\hline \hline
\end{tabular}

Table 10: Vector dimensions 